\title{
THE RIEMANN PROBLEM NEAR A HYPERBOLIC SINGULARITY II*
}

\author{
E. ISAACSON $\dagger$ AND B. TEMPLE $\ddagger$
}

\begin{abstract}
This paper is interested in classifying the solutions of Riemann problems for the $2 \times 2$ conservation laws that have homogeneous quadratic flux functions. Such flux functions approximate an arbitrary $2 \times 2$ system in a neighborhood of an isolated point where strict hyperbolicity fails. Here the solution for the symmetric systems in Region II of the four region classification of Schaeffer and Shearer is given. The solution is based on the qualitative shape of the integral curves described by Schaeffer and Shearer and a numerical calculation of the Hugoniot loci and their shock types.
\end{abstract}

Key words. Riemann problem, nonstrictly hyperbolic conservation laws, umbilic points

AMS(MOS) subject classifications. 65M10, 76N99, 35L65, 35L67

1. Introduction. This is the third in a series of papers [5], [6] in which we give the solution of the Riemann problem for quadratic conservation laws

$$
\begin{aligned}
& u_{t}+\frac{1}{2}\left\{a_{1} u^{2}+2 b_{1} u v+c_{1} v^{2}\right\}_{x}=0, \\
& v_{t}+\frac{1}{2}\left\{a_{2} u^{2}+2 b_{2} u v+c_{2} v^{2}\right\}_{x}=0,
\end{aligned}
$$

with initial data

$$
\mathbf{u}(x, 0)= \begin{cases}\mathbf{u}_{L} \equiv\left(u_{L}, v_{L}\right), & x<0 \\ \mathbf{u}_{R} \equiv\left(u_{R}, v_{R}\right), & x>0\end{cases}
$$

where $\mathbf{u} \equiv(u, v)$. Such conservation laws approximate a general $2 \times 2$ system of conservation laws in a neighborhood of an isolated point at which strict hyperbolicity fails. We use the normal form

$$
\begin{aligned}
& u_{1}+\frac{1}{2}\left\{a u^{2}+2 b u v+v^{2}\right\}_{x}=0, \\
& v_{1}+\frac{1}{2}\left\{b u^{2}+2 u v\right\}_{x}=0
\end{aligned}
$$

of Schaeffer and Shearer [13]. We let $\lambda_{1}(\mathbf{u}) \leqq \lambda_{2}(\mathbf{u})$ denote the eigenvalues of system (1) and note that $\lambda_{1}=\lambda_{2}$ only when $\mathbf{u}=\mathbf{0}$ in which case $\lambda=0$.

In the previous papers [5], [6] we presented the solution of the Riemann problem for the symmetric systems (1) in Regions III and IV which correspond to the parameter ranges

and

$$
1<a<2, \quad b=0
$$

$$
a>2, \quad b=0,
$$

* Received by the edtiors December 15, 1986; accepted for publication June 12, 1987.

+ Department of Mathematics, University of Wyoming, Laramie, Wyoming 82071. The work of this author was supported in part by National Science Foundation grant DMS-831229, Department of Energy contract DE-AC02-76ER03077, University of Wyoming Division of Basic Research, Air Force Office of Scientific Research grant AFOSR-85-0117 and CNPq/Brazil grant 403039/84-MA.

\$Department of Mathematics, University of California, Davis, California 95616 and Department of Mathematics and the Mathematics Research Center, University of Wisconsin-Madison, Madison, Wisconsin 53705. The work of this author was supported in part by FINEP/Brazil grant 4.3.82.017.9, CNPq/Brazil grant 1.01.10.011/84-ACI, National Science Foundation grant 527428INT-8415209, the U.S. Army under contract DAAG29-80-C-0041 and is based upon work supported by the National Science Foundation grants DMS-8210950, Mod. 1 and DMS-8210950, Mod. 4. 
respectively. In the present work we give the solution of the Riemann problem for the parameter range

$$
0<a<1, \quad b=0
$$

which corresponds to the symmetric systems in Region II. The solutions consist of (portions of) the 1-wave curves $\mathscr{W}_{1}(\mathbf{u})$ and the 2 -wave curves $\mathscr{W}_{2}(\mathbf{u})$. These are obtained from the Hugoniot loci and integral curves by means of both numerical and analytical evidence. Our constructions indicate the following result.

THEOREM. For each pair of states $\mathbf{u}_{L}$ and $\mathbf{u}_{R}$, the solution of the Riemann problem consists of a succession of 1-waves and 2-waves from $\mathbf{u}_{L}$ to $\mathbf{u}_{R}$. Moreover, the solution is unique in $x, t$-space, depends continuously on $\mathbf{u}_{L}$ and $\mathbf{u}_{R}$, and all shocks appearing in solutions are 1-shocks or 2-shocks in the sense of Lax [10].

We refer to [5] for a detailed discussion of the problem and the notation.

As in [5], [6] we obtain the shapes and shock types of Hugoniot loci $\mathscr{H}\left(\mathbf{u}_{L}\right)$ by means of numerical calculation. This, together with the qualitative features of the integral curves given in [13], [2] (see Fig. 1), is the basis for our construction of the solutions. We present the solution of the Riemann problem in a series of diagrams for representative values of $\mathbf{u}_{L}$.

The qualitative features of the solution diagrams change precisely when $\mathbf{u}_{L}$ crosses either the Hugoniot locus $\mathscr{H}(\mathbf{0})$ or a ray $\lambda_{1}=0$. In Region II, the Hugoniot locus $\mathscr{H}(\mathbf{0})$ consists of three straight lines through the origin [13]; we refer to them as axes. For the symmetric systems in Region II, one of the axes is the $u$-axis and the other two are given by

$$
v= \pm \sqrt{2-a} u
$$

The rays $\lambda_{1}=0$ are given by

$$
v= \begin{cases}-\sqrt{a} u & \text { in the lower half plane } \\ +\sqrt{a} u & \text { in the upper half plane. }\end{cases}
$$

We give the solution of the Riemann problem for representative values of $\mathbf{u}_{L}$ in the lower half plane $(v \leqq 0)$ because of the symmetries in system (1) (see [5]). Since the solutions change qualitatively only at the rays $\mathscr{H}(\mathbf{0})$ and $\lambda_{1}=0$, we choose a representative value of $\mathbf{u}_{L}$ from each interior and boundary ray of the sectors (see Fig. 2)

$$
\begin{aligned}
& \mathscr{A}_{1} \equiv\left\{\mathbf{u}_{L}: \theta_{*}<\theta<0\right\}, \\
& \mathscr{A}_{2} \equiv\left\{\mathbf{u}_{L}: \theta_{23}<\theta<\theta_{*}\right\}, \\
& \mathscr{A}_{3} \equiv\left\{\mathbf{u}_{L}: \theta_{34}<\theta<\theta_{23}\right\}, \\
& \mathscr{A}_{4} \equiv\left\{\mathbf{u}_{L}:-\pi<\theta<\theta_{34}\right\},
\end{aligned}
$$

where

$$
\begin{aligned}
& \theta_{*} \equiv \arctan (-\sqrt{a}), \\
& \theta_{23} \equiv \arctan (-\sqrt{2-a}), \\
& \theta_{34} \equiv \arctan (+\sqrt{2-a})-\pi .
\end{aligned}
$$

The angles correspond to the ray $\lambda_{1}=0$ and the axes, respectively.

A new feature in Region II is that both the 1-wave curve $\mathscr{W}_{1}\left(\mathbf{u}_{L}\right)$ and the 2-wave curve $\mathscr{W}_{2}\left(\mathbf{u}_{L}\right)$ may be disconnected. In [5], [6], the Riemann problem solution is depicted in diagrams by a connected path of wave curves in the $u, v$-plane from $\mathbf{u}_{L}$ to $\mathbf{u}_{R}$. 


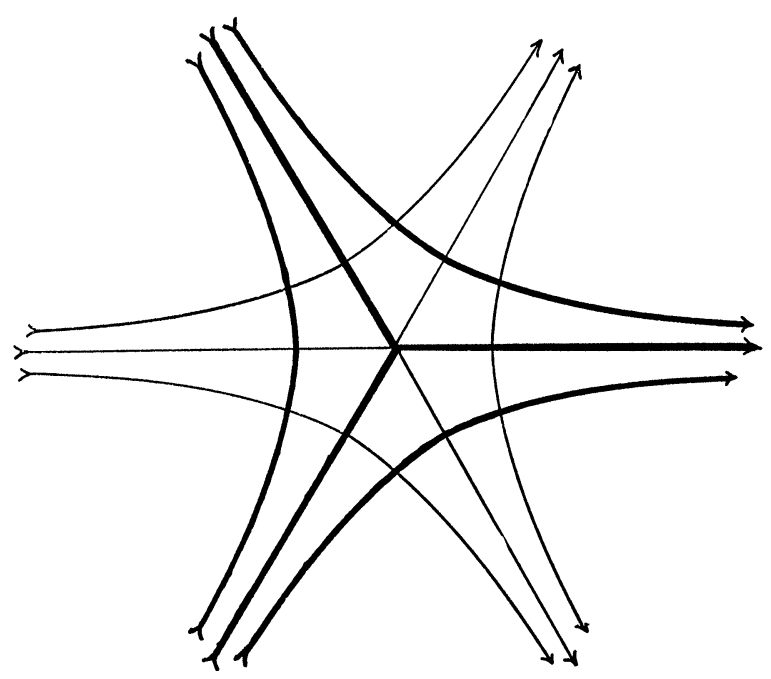

FIG. 1. Integral curves.

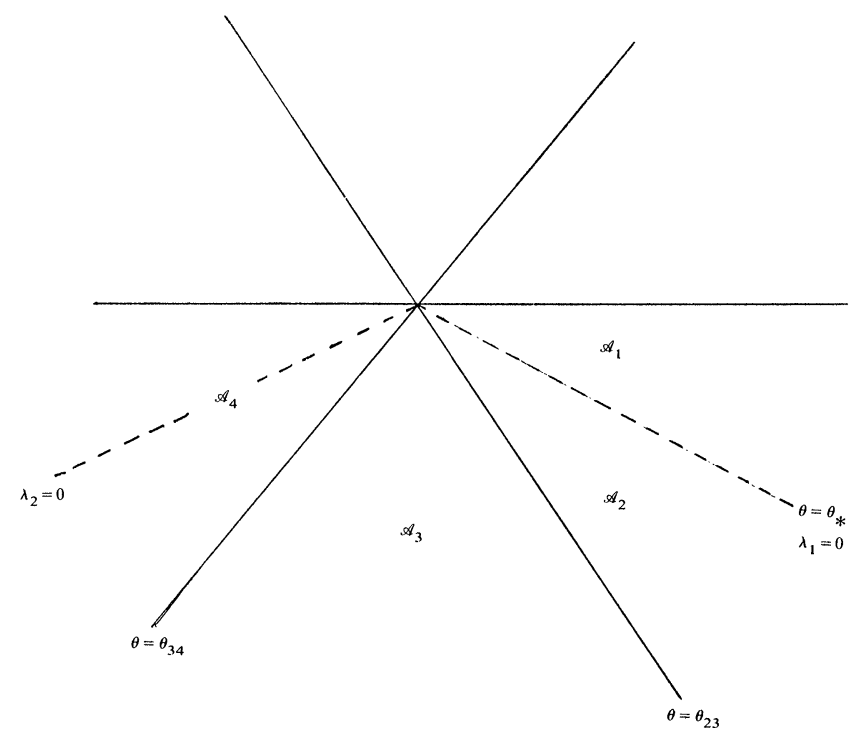

FIG. 2. The sectors $\mathscr{A}_{1}-\mathscr{A}_{4}$ and their boundaries. 
However in the present case, for states $\mathbf{u}_{L}$ in sectors $\mathscr{A}_{1}, \mathscr{A}_{2}$ and $\mathscr{A}_{3}$, there is no such connected path of wave curves whenever the state $\mathbf{u}_{R}$ lies in a certain triangular region of the $u, v$-plane. We address this issue further in the next section.

2. Remarks. The integral curves for the symmetric systems (1), (2) in Region II are depicted in Fig. 1 (see [13], [2]). The Hugoniot loci for the representative values of $\mathbf{u}_{L}$ chosen above are depicted in Figs. 3(a)-(j). The shock types are also indicated (see $\S 2.1$ ). Note that in Fig. 3(e) the disconnected component of the Hugoniot locus $\mathscr{H}\left(\mathbf{u}_{L}\right)$ contains a segment consisting of 1 -shocks. This phenomenon was already seen in Region III [6]. A new feature in Region II is that the disconnected component of $\mathscr{H}\left(\mathbf{u}_{L}\right)$ also contains a segment consisting of 2-shocks. It is this feature that leads to the appearance of the "triangular" region in Figs. $4\left(c_{3}\right)-(g)$.

We now discuss the solution diagrams in Figs. $4(a)-(j)$. The solution consists of a 1-composite wave followed by a 2-composite wave as in [5], [6]. Each diagram depicts the Riemann problem solution for a fixed left state $\mathbf{u}_{L}$. An arbitrary point in the diagram represents $\mathbf{u}_{R}$. To obtain the solution of the Riemann problem, follow the arrows from $\mathbf{u}_{L}$ to $\mathbf{u}_{R}$ along the 1- and 2-wave curves. Clarification of this procedure is required in Figs. $4\left(c_{3}\right)-(g)$ due to the appearance of the triangular region of disconnected 2-shocks; in fact, we must augment this procedure when either: (1) $\mathbf{u}_{R}$ lies in the triangular region; or (2) the 2-wave back from $\mathbf{u}_{R}$ intersects the boundary of the region. To illustrate, we describe in more detail the solutions in Fig. $4\left(\mathrm{e}_{1}\right)$ in which the region of disconnected 2-shocks is the triangle CEF. The dashed line segments between the vertex $C$ and the base $E F$ in triangle $C E F$ are the 2-shock portions of the disconnected components of $\mathscr{H}(\mathbf{u})$ for $\mathbf{u}$ between $\mathbf{u}_{*}$ and $H$ on $\mathscr{W}_{1}\left(\mathbf{u}_{L}\right)$. In particular, $B D$ is the disconnected 2-shock portion of $\mathscr{H}\left(\mathbf{u}_{L}\right)$; segments between $C$ and $B D$ are 2-shock portions of Hugoniot loci for states between $\mathbf{u}_{*}$ and $\mathbf{u}_{L}$; and segments between $B D$ and $E F$ are 2-shock portions of Hugoniot loci for states between $\mathbf{u}_{L}$ and $H$. Thus, for $\mathbf{u}_{R}$ in the region $C E F$, the solution of the Riemann problem consists of a 1-wave from $\mathbf{u}_{L}$ to an intermediate state $\mathbf{u}_{M}$ (between $H$ and $\mathbf{u}_{*}$ ) followed by a 2-shock from $\mathbf{u}_{M}$ to $\mathbf{u}_{R}$. If $\mathbf{u}_{R}$ lies on any of the 2-rarefaction curves emanating from side $C F$, then the Riemann problem solution consists of the solution from $\mathbf{u}_{L}$ to the appropriate state on the side $C F$ followed by a 2-rarefaction wave from that state to $u_{\mathrm{R}}$. For completeness, we indicate in Fig. $4\left(e_{2}\right)$ the regions of $\mathbf{u}_{R}$ in which the Riemann problem solutions have a given wave structure.

2.1. Legend. $\rightarrow$ 1-rarefaction; $\longrightarrow$ 1-expansive shock; ---1 -shock, $\rightarrow \longrightarrow$ 2-rarefaction; $=2$-expansive shock; $--\rightarrow--2$-shock (with or without arrows); $\rightarrow$ 1-composite (rarefaction followed by shock at characteristic speed-; - $\bigcirc-$ compressive shock; $-\times-$ crossing shock; $-\square-$ expansive shock; -2 -boundary, triple shock curves; - Hugoniot locus of $\mathbf{u}_{L}$ and $u$-axis.

Note 1. Arrows on rarefaction curves indicate the direction of increasing eigenvalue. Arrows on shock curves indicate the direction of decreasing shock speed.

Note 2. 1- and 2-shocks in the Hugoniot locus of $\mathbf{u}_{L}$ are indicated by dashed lines supported by the solid line for the Hugoniot locus. 


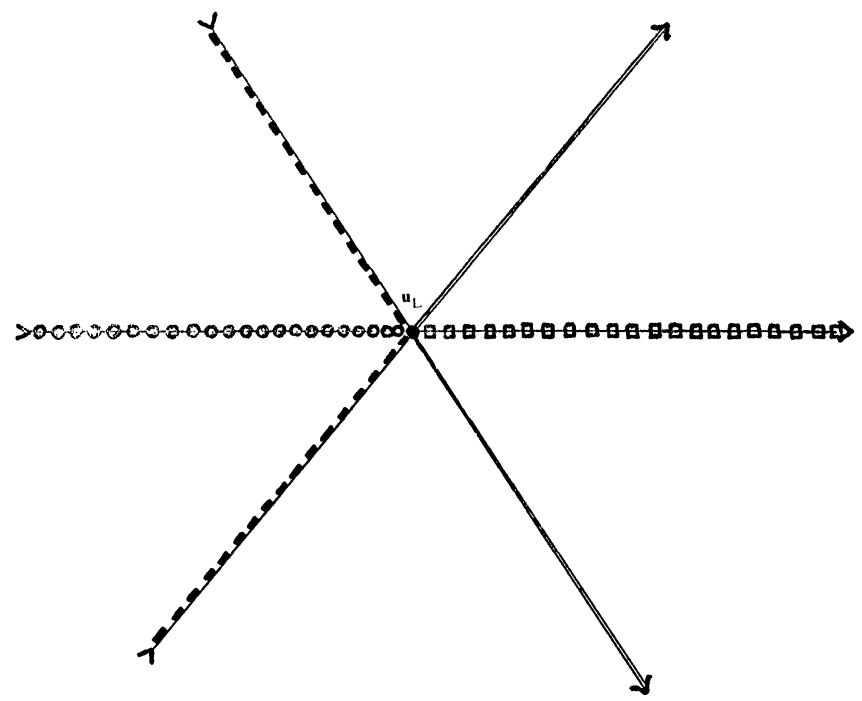

FIG. 3(a). The Hugoniot locus $\mathscr{H}\left(\mathbf{u}_{L}\right)$ for $\mathbf{u}_{L}=\mathbf{0}$.

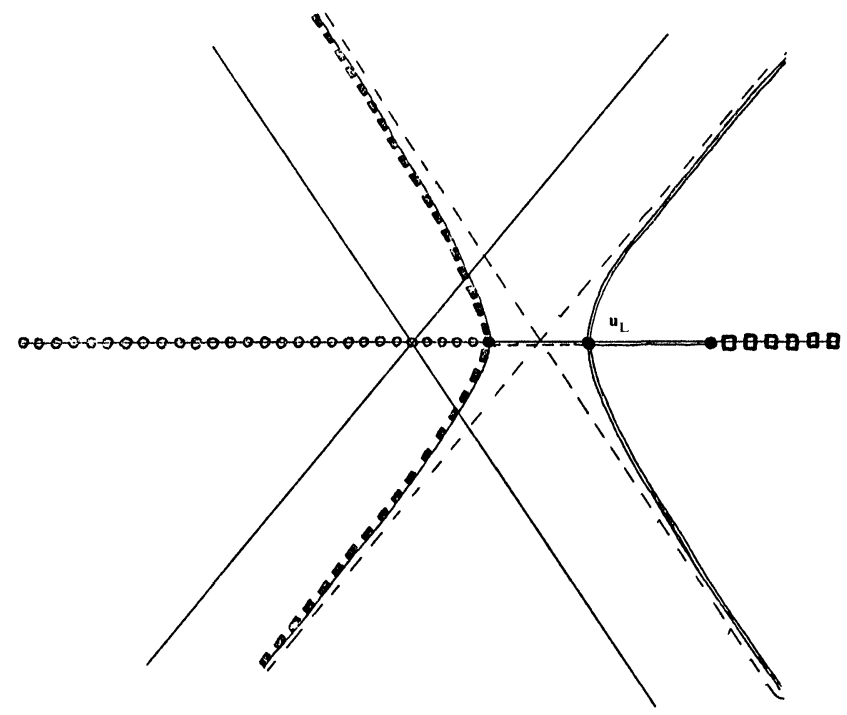

FIG. 3(b). The Hugoniot locus $\mathscr{H}\left(\mathbf{u}_{L}\right)$ for $\theta_{L}=0$. 
QUADRATIC RIEMANN PROBLEMS III

1307

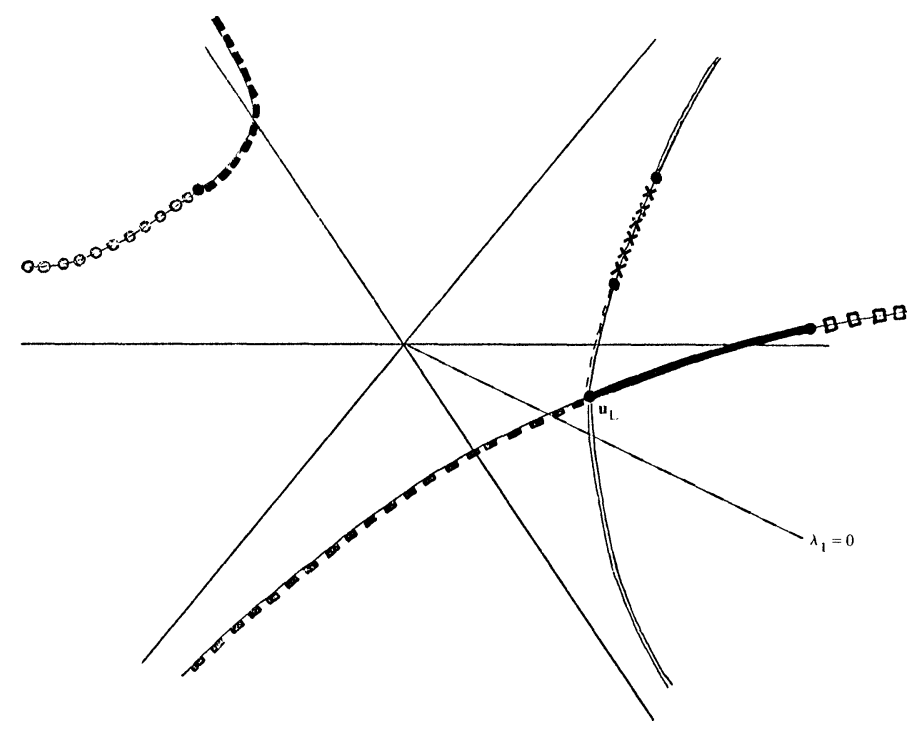

FIG. 3(c). The Hugoniot locus $\mathscr{H}\left(\mathbf{u}_{L}\right)$ for $\mathbf{u}_{L} \in \mathscr{A}_{1}$.

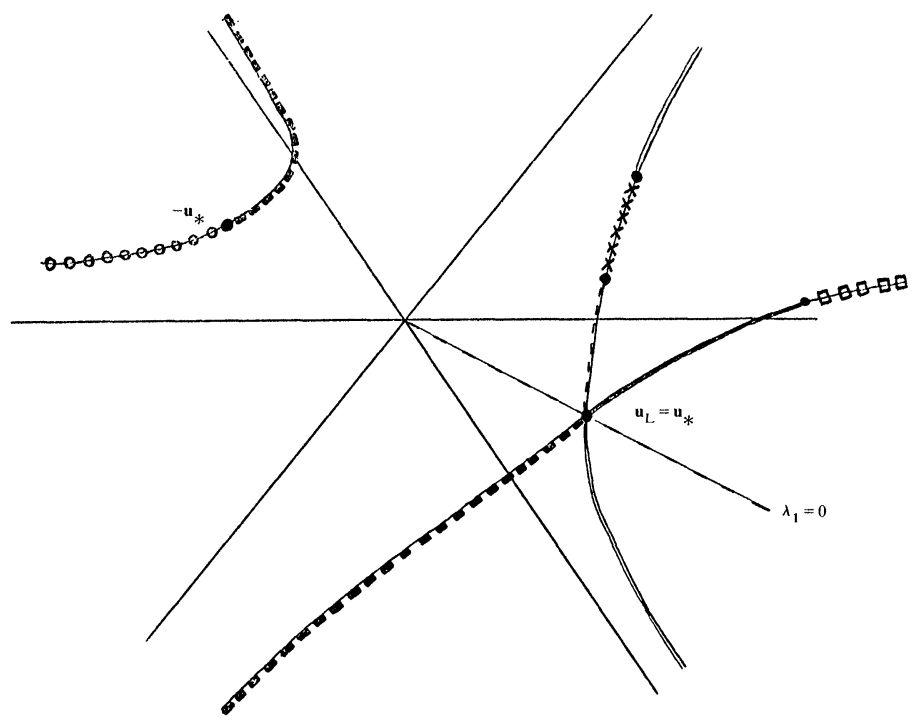

FIG. 3(d). The Hugoniot locus $\mathscr{H}\left(\mathbf{u}_{L}\right)$ for $\theta_{L}=\theta_{*}$. 


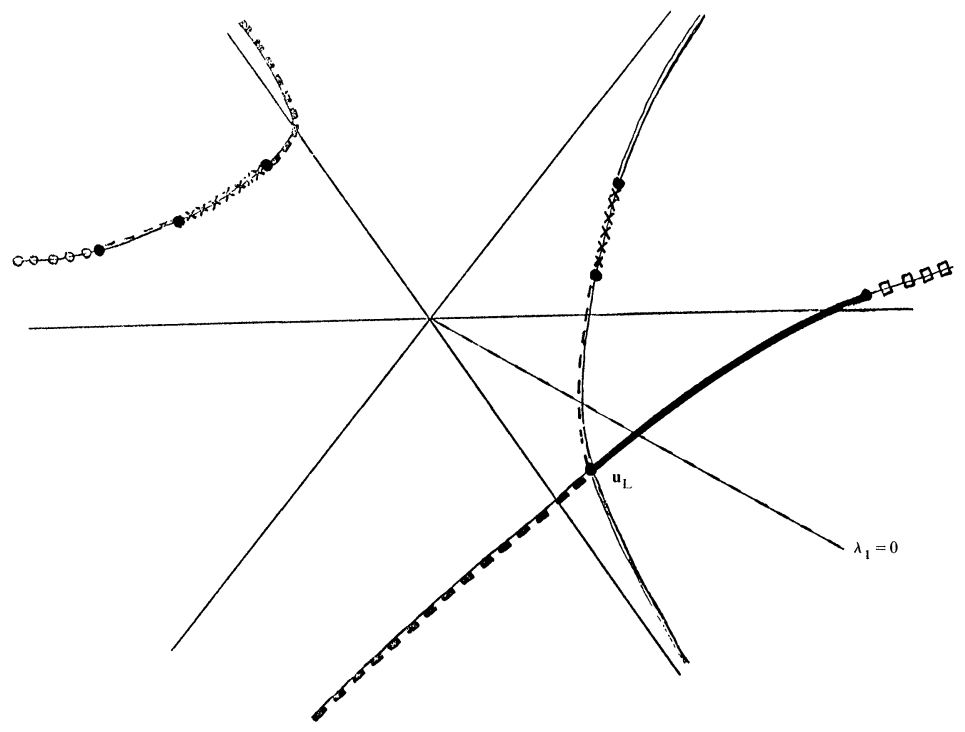

FIG. 3(e). The Hugoniot locus $\mathscr{H}\left(\mathbf{u}_{L}\right)$ for $\mathbf{u}_{L} \in \mathscr{A}_{2}$.

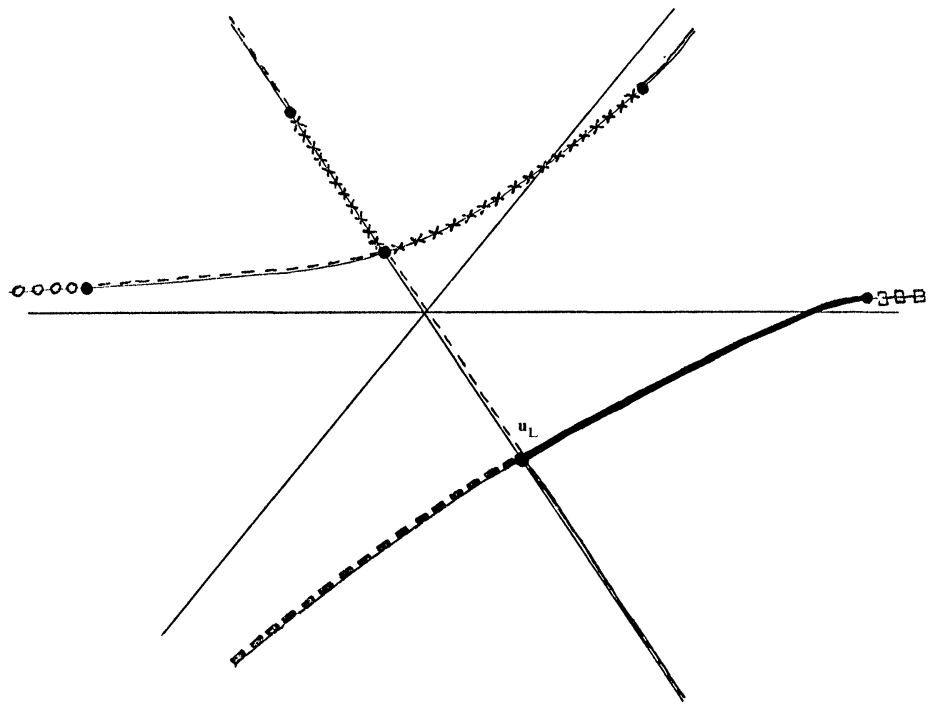

FIG. 3(f). The Hugoniot locus $\mathscr{H}\left(\mathbf{u}_{L}\right)$ for $\theta_{L}=\theta_{23}$. 


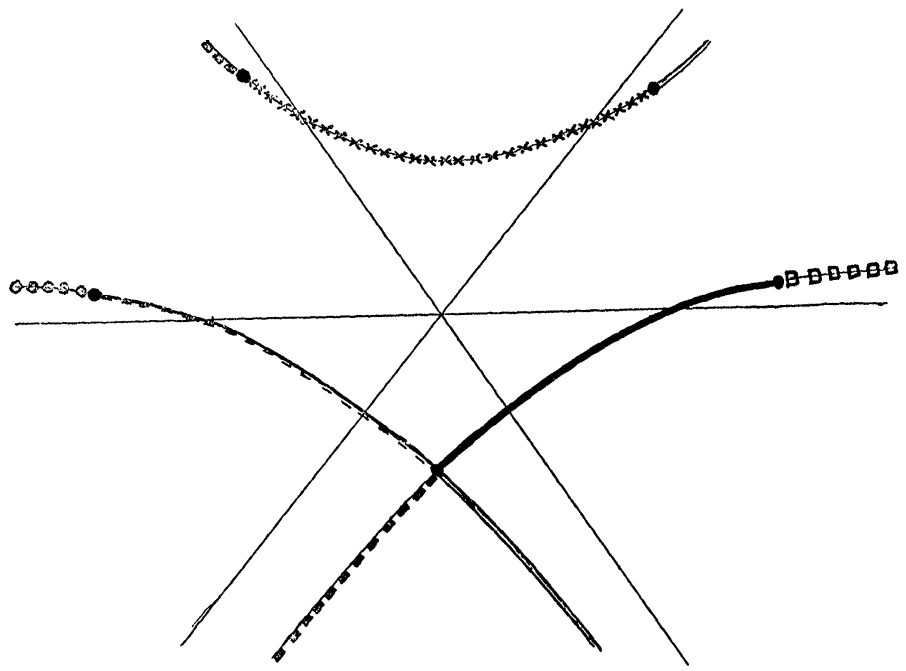

FIG. 3(g). The Hugoniot locus $\mathscr{H}\left(\mathbf{u}_{L}\right)$ for $\mathbf{u}_{L} \in \mathscr{A}_{3}$.

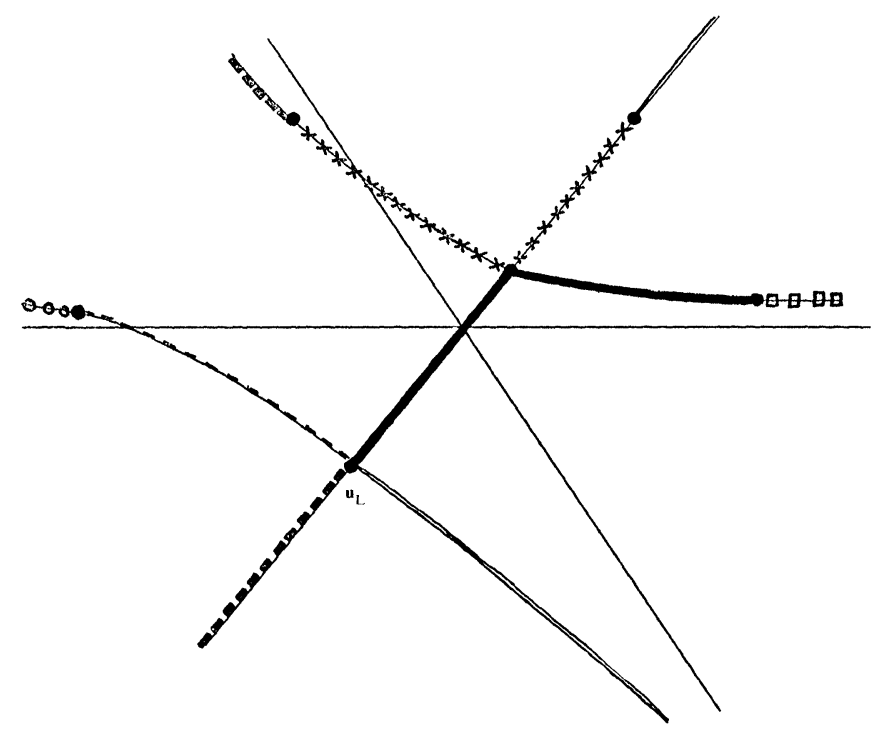

FIG. 3(h). The Hugoniot locus $\mathscr{H}\left(\mathbf{u}_{I}\right)$ for $\theta_{l}=\theta_{34}$. 


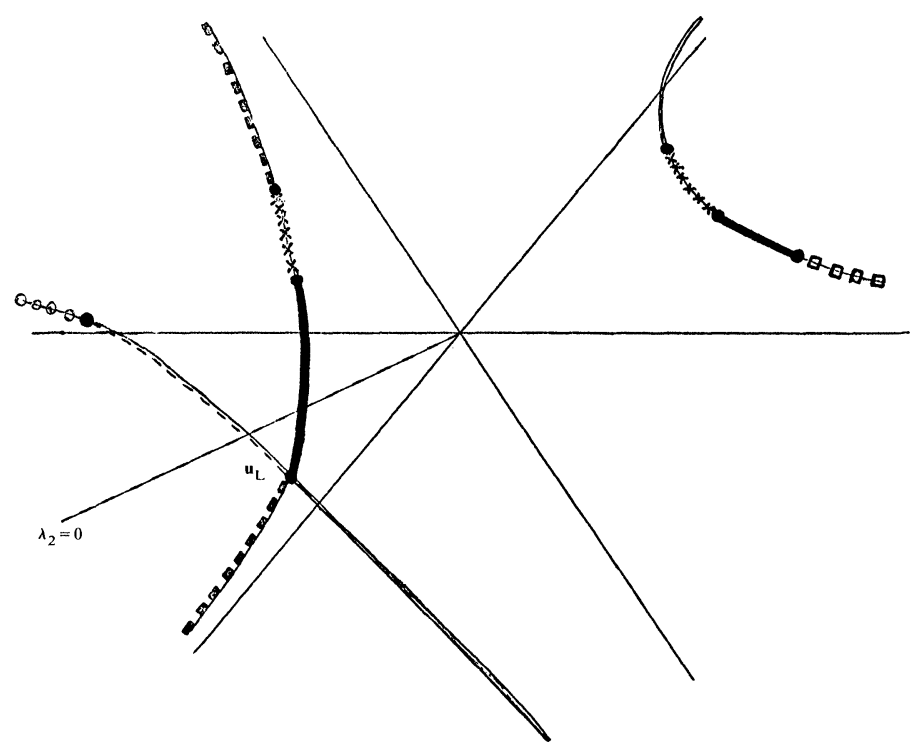

FIG. 3(i). The Hugoniot locus $\mathscr{H}\left(\mathbf{u}_{L}\right)$ for $\mathbf{u}_{L} \in \mathscr{A}_{4}$.

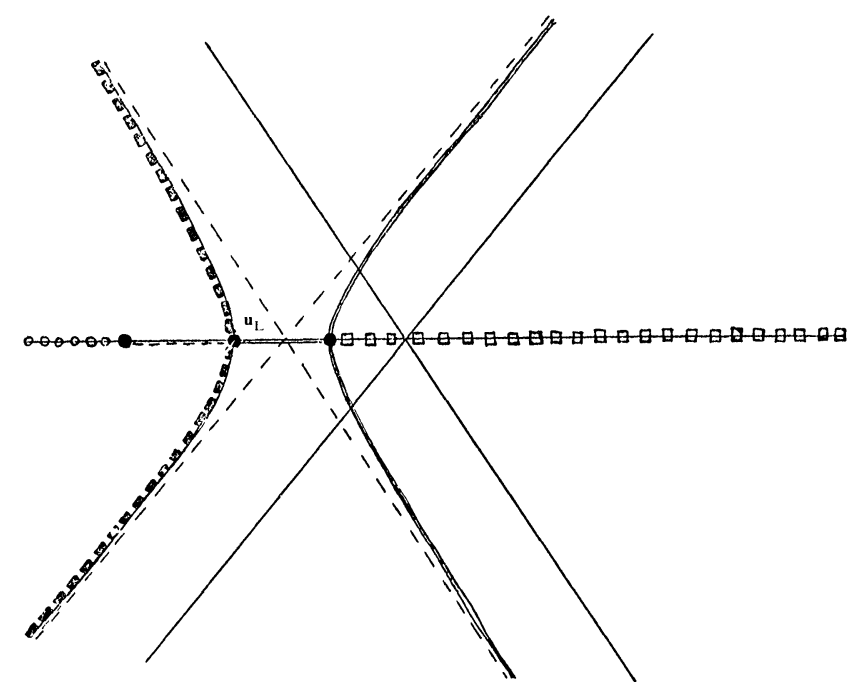

FIG. 3(j). The Hugoniot locus $\mathscr{H}\left(\mathbf{u}_{L}\right)$ for $\theta_{L}=-\pi$. 


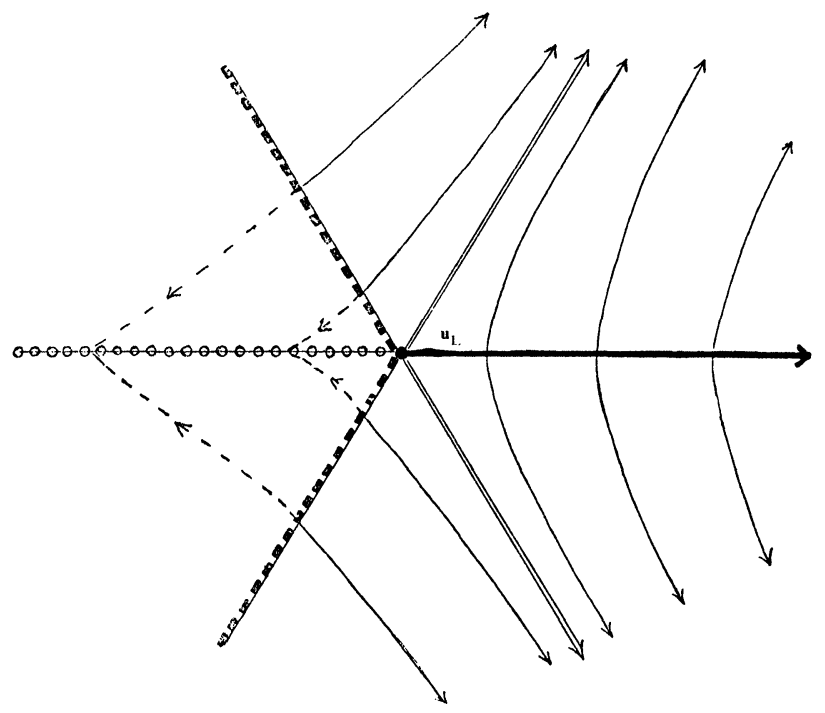

FIG. 4(a). Solution diagram for $\mathbf{u}_{L}=\mathbf{0}$.

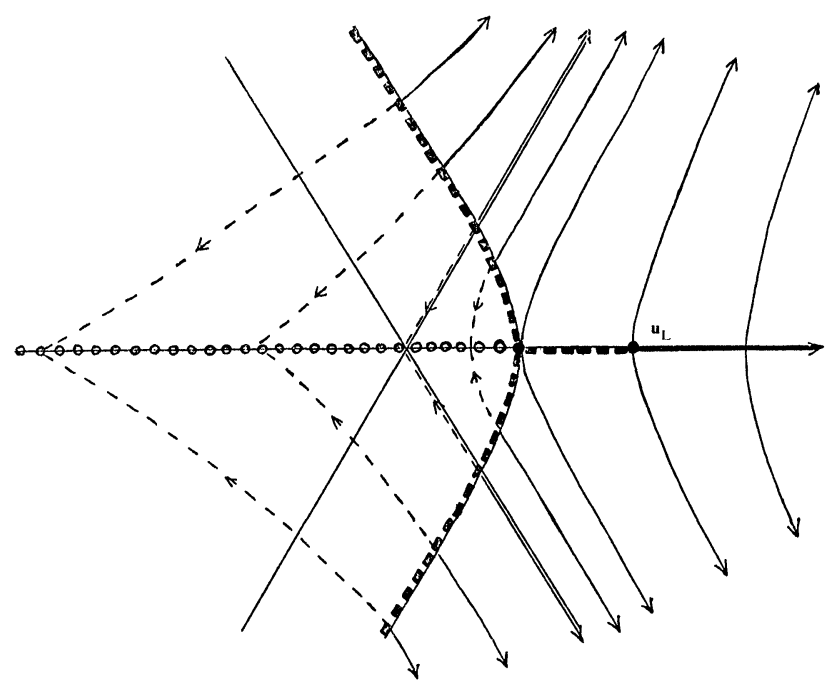

FIG. 4(b). Solution diagram for $\theta_{L}=0$. 


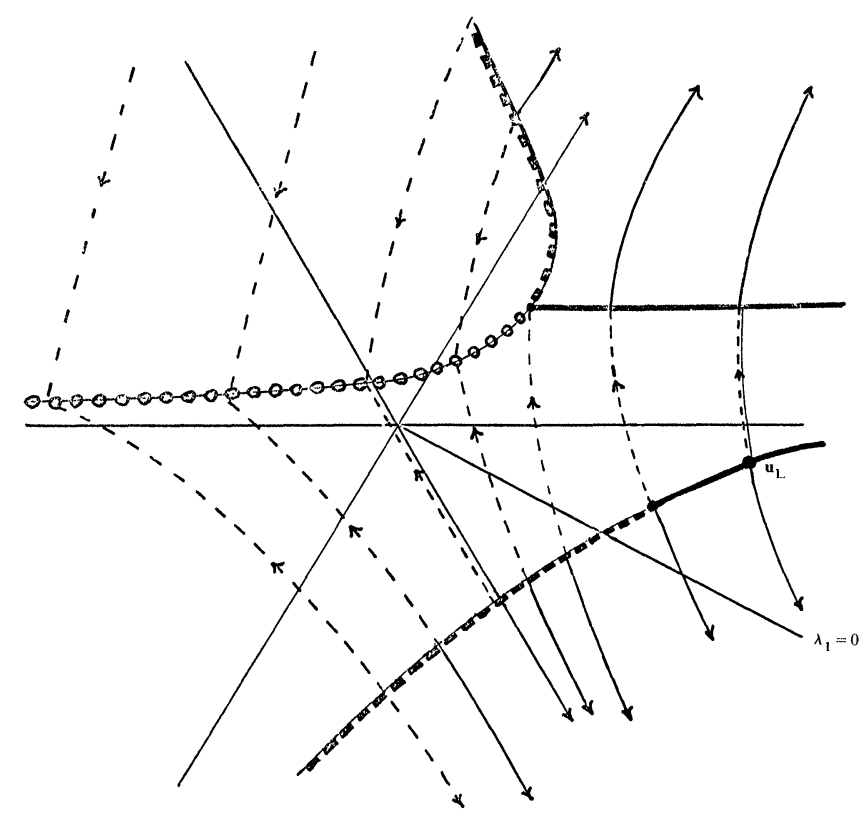

FIG. $4\left(c_{1}\right)$. Solution diagram for $\mathbf{u}_{L} \in \mathscr{A}_{1}$.

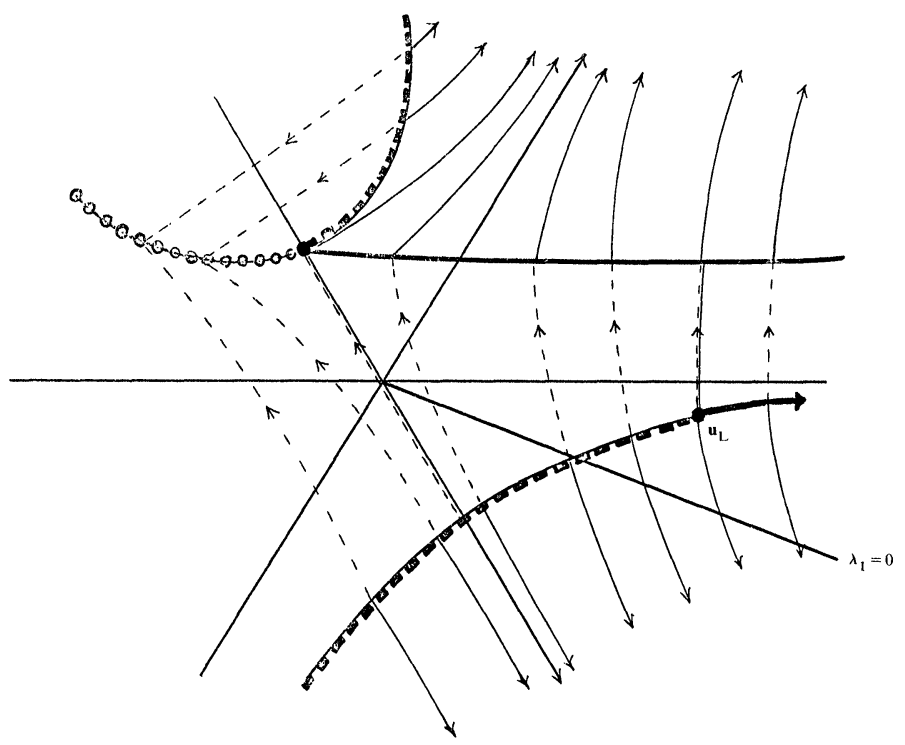

FIG. $4\left(\mathrm{c}_{2}\right)$. Solution diagram for $\mathbf{u}_{L} \in \mathscr{A}_{1}$. 


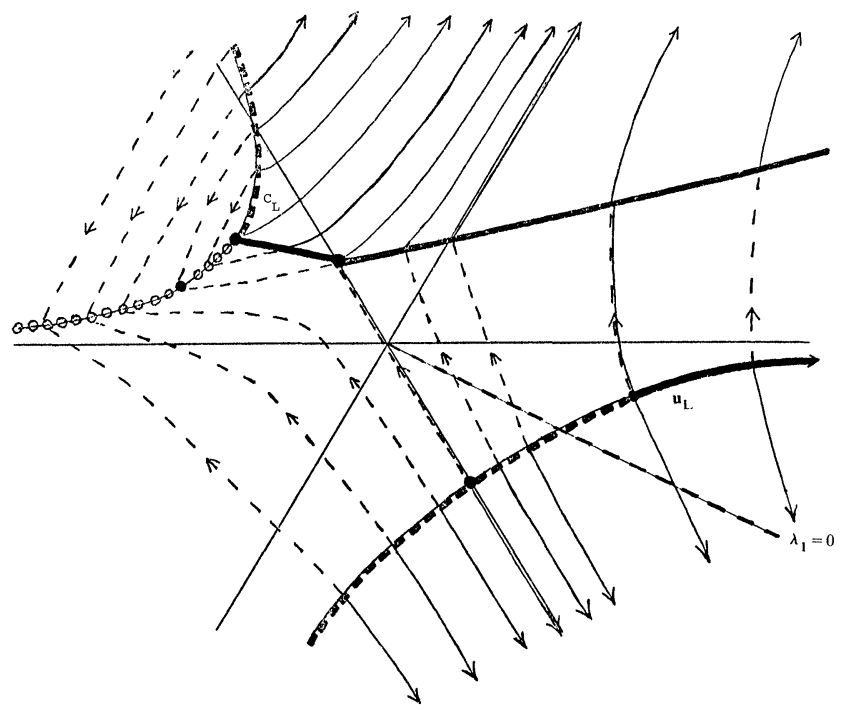

FIG. $4\left(c_{3}\right)$. Solution diagram for $\mathbf{u}_{L} \in \mathscr{A}_{1}$.

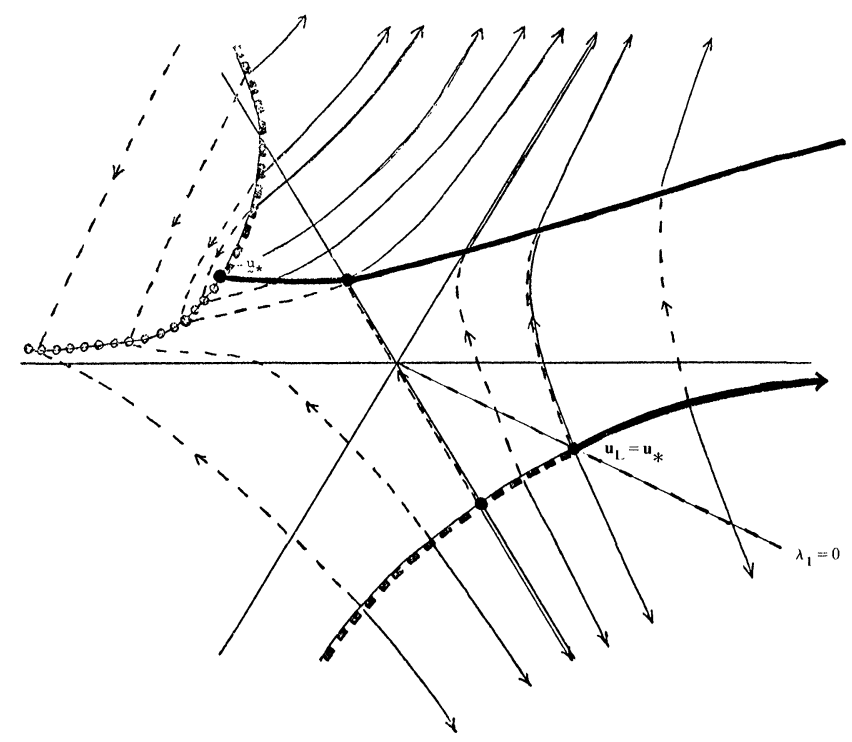

FIG. 4(d). Solution diagram for $\theta_{L}=\theta_{*}$. 


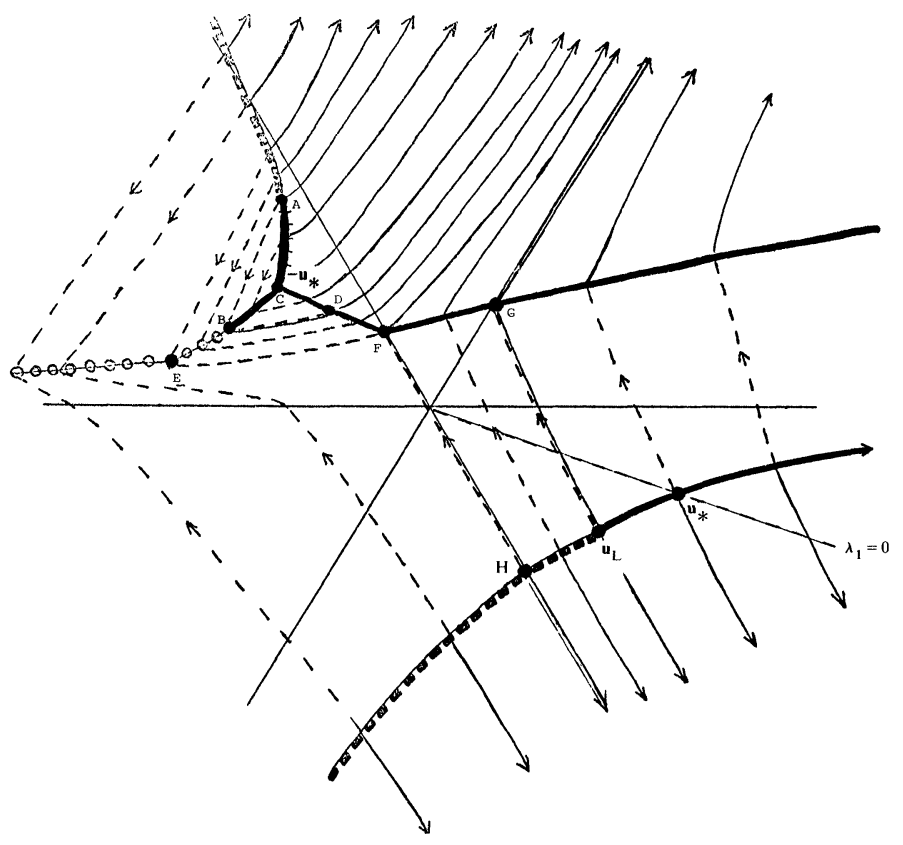

FIG. $4\left(\mathrm{e}_{1}\right)$. Solution diagram for $\mathbf{u}_{L} \in \mathscr{A}_{2}$.

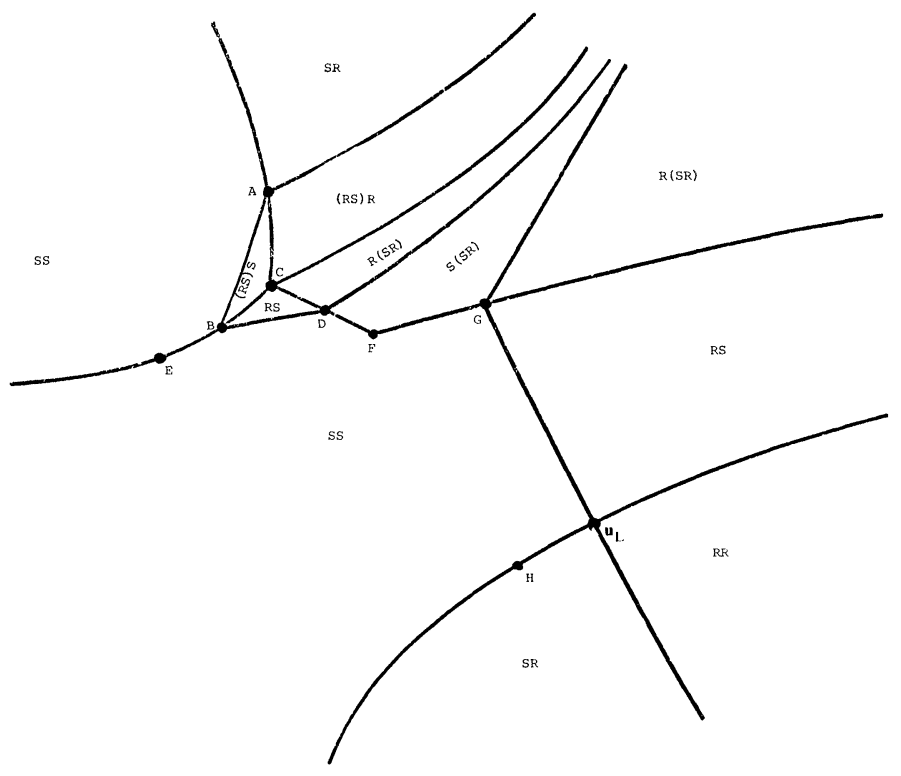

FIG. 4(e $\left.e_{2}\right)$. Wave structure for Fig. $4\left(\mathrm{e}_{1}\right)$. 


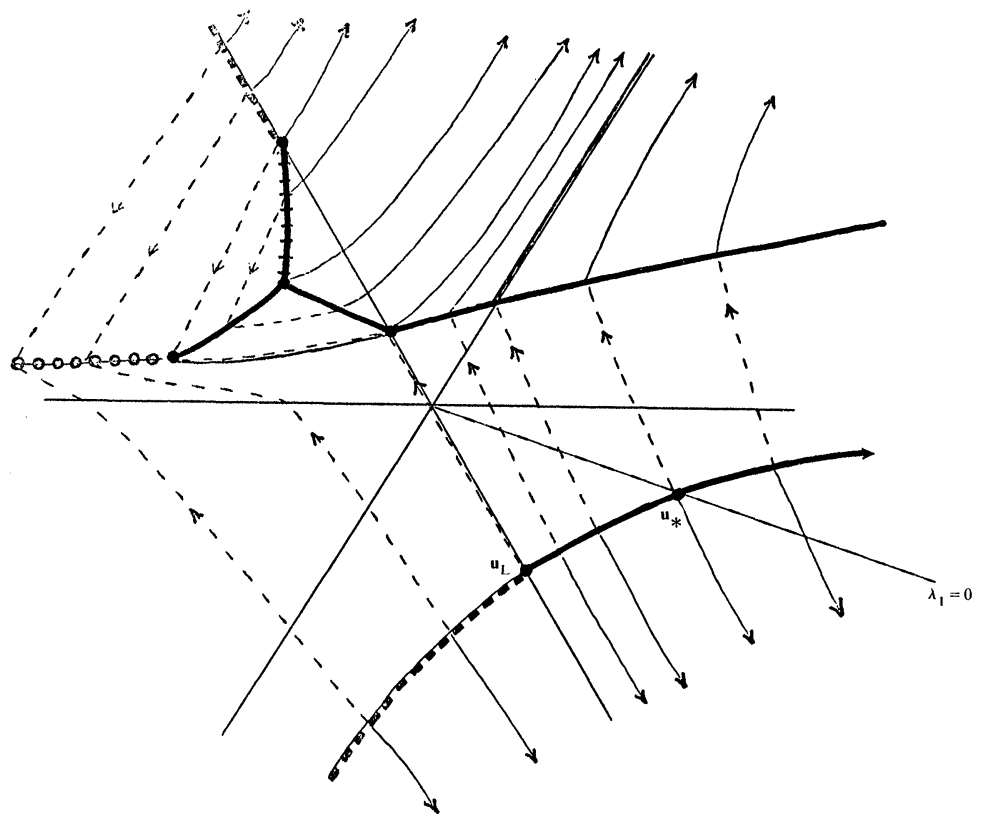

FIG. 4(f). Solution diagram for $\theta_{L}=\theta_{23}$.

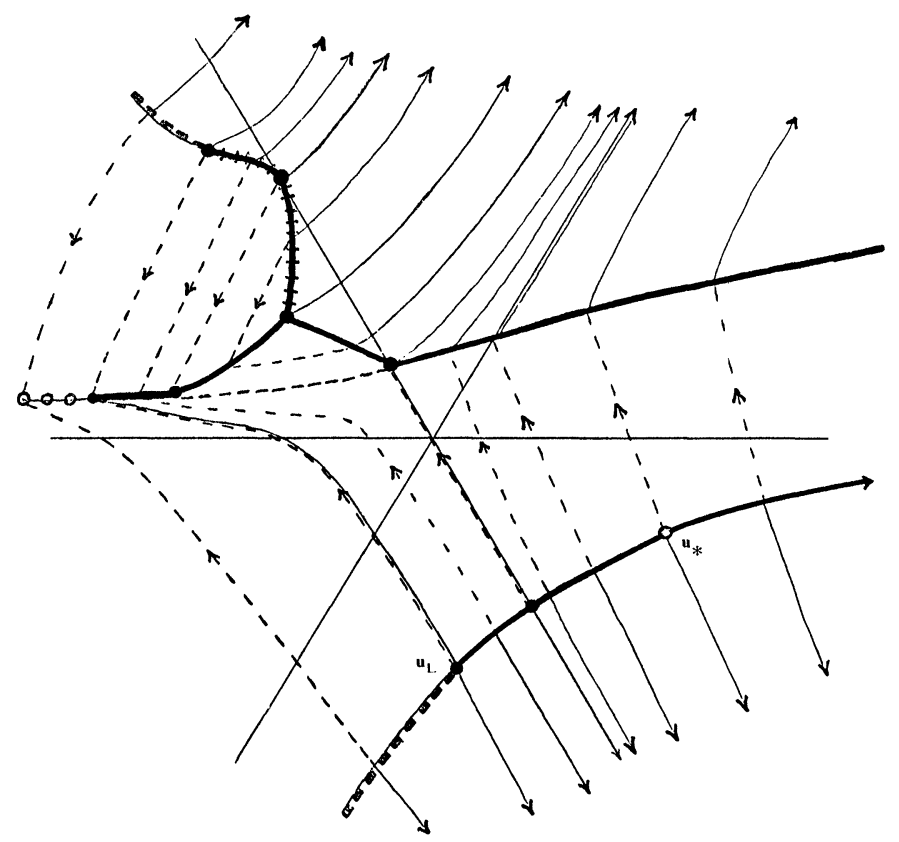

FIG. 4(g). Solution diagram for $\mathbf{u}_{L} \in \mathscr{A}_{3}$. 


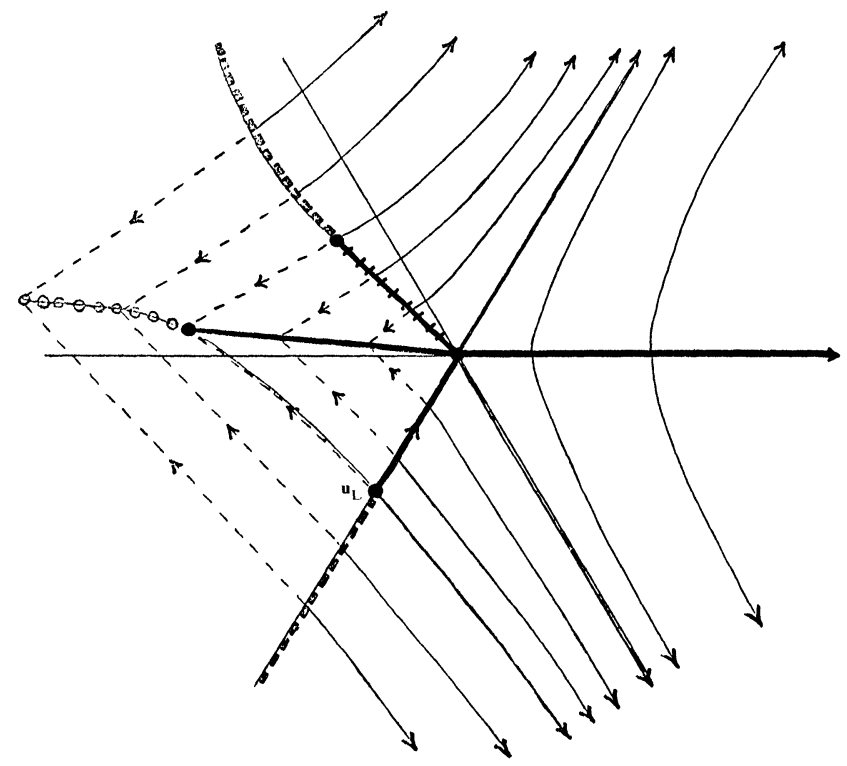

FIG. 4(h). Solution diagram for $\theta_{L}=\theta_{34}$.

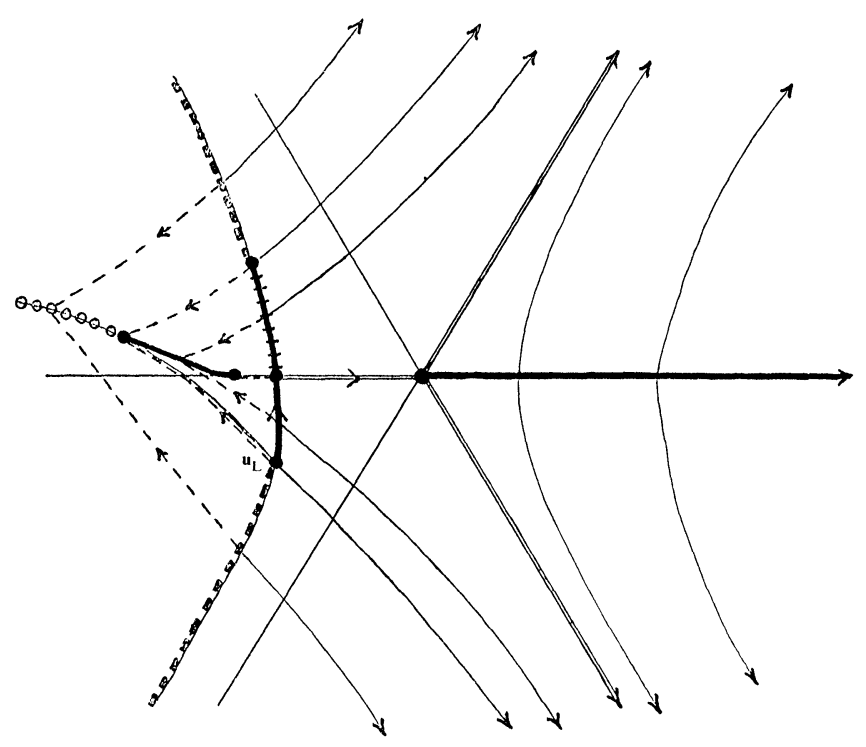

FIG. 4(i). Solution diagram for $\mathbf{u}_{L} \in \mathscr{A}_{4}$. 


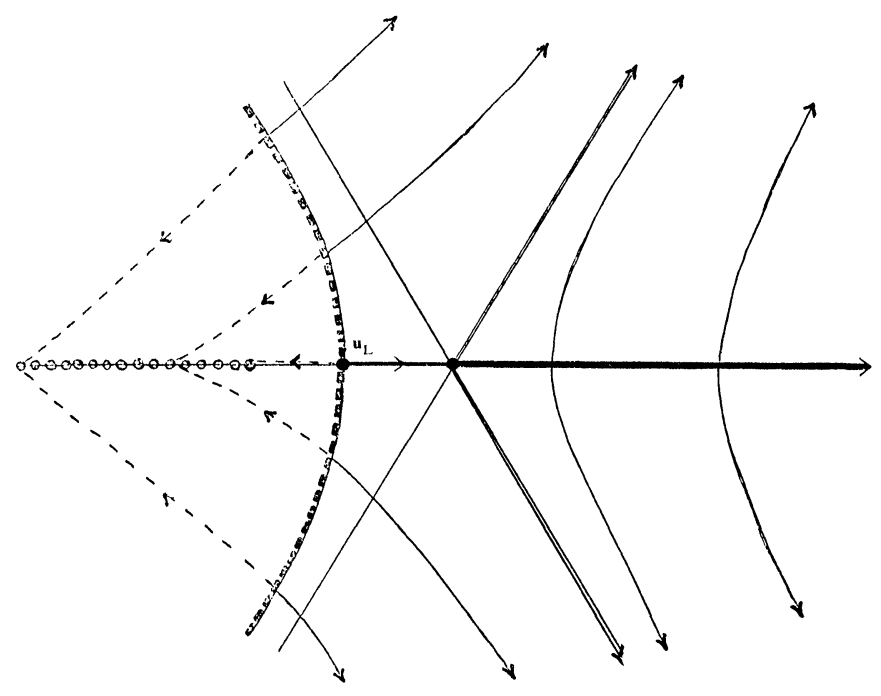

FIG. 4(j). Solution diagram for $\theta_{L}=-\pi$.

Acknowledgments. We wish to acknowledge the assistance of Dan Marchesin and Bradley Plohr with the numerical aspects of the problem. We also thank David Schaeffer and Michael Shearer for helpful discussions. Finally, we would like to thank Dan and Paulo Paes-Leme for their gracious hospitality during our stay in Brazil.

\section{REFERENCES}

[1] R. Courant And K. O. Friedrichs, Supersonic Flow and Shock Waves, John Wiley, New York, 1948.

[2] G. Darboux, Théorie Générale des Surfaces, Chelsea, New York, 1972.

[3] E. IsAACSON, Global solution of a Riemann problem for a non-strictly hyperbolic system of conservation laws arising in enhanced oil recovery, J. Comp. Phys., to appear.

[4] E. IsAacson AND B. Temple, Analysis of a singular hyperbolic system of conservation laws, J. Differential Equations, 65 (1986), pp. 250-268.

[5] E. IsaAcson, D. Marchesin, B. Plohr, And B. Temple, The Riemann problem near a hyperbolic singularity I, SIAM J. Appl. Math., 48 (1988), pp. 1009-1032

[6] E. ISAACSON AND B. TEMPLE, The Riemann problem near a hyperbolic singularity II, SIAM J. Appl. Math., this issue, pp. 1287-1301.

[7] — Examples and classification of non-strictly hyperbolic systems of conservation laws, Abstracts of AMS, January 1985; Presented in the Special Session on Non-Strictly Hyperbolic Conservation Laws at the Winter Meeting of the Amer. Math. Soc., Anaheim, January 1985.

[8] B. KEYFITZ AND H. KRANZER, A system of non-strictly hyperbolic conservation laws arising in elasticity theory, Arch. Rational Mech. Anal., 72 (1980), pp. 219-241.

[9] - The Riemann problem for a class of conservation laws exhibiting a parabolic degeneracy, J. Differential Equations, 47 (1983), pp. 35-65.

[10] P. D. LAX, Hyperbolic systems of conservation laws, II, Comm. Pure Appl. Math., 19 (1957), pp. 537-566.

[11] - Shock waves and entropy, in Contributions to Nonlinear Functional Analysis, E. H. Zarantonello, ed., Academic Press, New York, 1971, pp. 643-654.

[12] T. P. LIU, The Riemann problem for general $2 \times 2$ conservation laws, Trans. Amer. Math. Soc., 199 (1974), pp. 89-112.

[13] D. G. SCHAEFFER AND M. SHEARER, The classification of $2 \times 2$ systems of non-strictly hyperbolic conservation laws, with application to oil recovery, with Appendix by D. Marchesin, P. J. Paes-Leme, D. G. Schaeffer, and M. Shearer, Comm. Pure Appl. Math., 40 (1987), pp. 141-178 
[14] M. Shearer, D. G. Schaeffer, D. Marchesin, And P. J. Paes-Leme, Solution of the Riemann problem for a prototype $2 \times 2$ system of non-strictly hyperbolic conservation laws, Arch. Rational Mech. Anal., 97 (1987), pp. 299-320.

[15] J. A. SMoller, Shock waves and reaction diffusion equations, Springer-Verlag, New York, Berlin, Heidelberg, 1982.

[16] B. TEMPLE, Global solution of the Cauchy problem for a class of $2 \times 2$ non-strictly hyperbolic conservation laws, Adv. in Appl. Math., 3 (1982), pp. 335-375.

[17] - Systems of conservation laws with coinciding shock and rarefaction curves, Contemp. Math., 17 (1983), pp. 143-151. 